\title{
Acute Right Ventricular Failure in a Patient with Non-ischemic Cardiogenic Shock on Left-Sided Mechanical Circulatory Support
}

\author{
Shuvodra Routh $^{1}$, Carly Fabrizio ${ }^{1}$, Christopher Sciortino ${ }^{1}$, Arman Kilic ${ }^{2}$, Catalin Toma ${ }^{2}$, \\ Raj Ramanan ${ }^{2}$, Jeffrey Fowler ${ }^{2}$, Parmjeet Randhawa ${ }^{2}$, and Gavin Hickey ${ }^{3}$ \\ ${ }^{1}$ University of Pittsburgh Medical Center \\ ${ }^{2}$ Affiliation not available \\ ${ }^{3}$ University of Pittsburgh Medical Center Health System
}

April 7, 2021

\begin{abstract}
We report a case of acute right ventricular failure in a patient with cardiogenic shock on left-sided mechanical circulatory support with Impella 5.0. The patient was successfully bridged to heart transplantation using additional right-sided support with ProtekDuo. Key learning points of the case include prompt recognition of right ventricular failure in patients on left-sided support, early consideration of right-ventricular mechanical support platforms, and timely deployment of right-sided mechanical support.
\end{abstract}

Title:

Acute Right Ventricular Failure in a Patient with Non-ischemic Cardiogenic Shock on Left-Sided Mechanical Circulatory Support

Running Head:

Acute RV Failure in a Patient on Left-Sided MCS

Authors:

Shuvodra Routh, MD

Carly Fabrizio, MD

Christopher M. Sciortino, MD PhD

Arman Kilic, MD

Catalin Toma, MD

Raj Ramanan, MD

Jeffrey A. Fowler, DO

Parmjeet S. Randhawa, MD

Gavin W. Hickey, MD

Affiliated institution of authors :

University of Pittsburgh Medical Center, 200 Lothrop Street, Pittsburgh, PA 15213 


\section{Total word count:}

1,427

Funding:

None

Conflicts of Interest:

None

IRB approval statement:

IRB approval statement is not applicable to this article as it does not involve research conducted on human subjects.

Informed consent statement:

Informed consent statement is not applicable to this article as all patient identities have been kept anonymous and protected health information has been kept confidential.

Data Availability Statement:

Data sharing is not applicable to this article as no new data were created or analyzed in this study.

Address for correspondence:

Shuvodra Routh, MD

200 Lothrop Street, N-715

Pittsburgh, PA 15213

E-mail: rouths@upmc.edu, Fax: 412-692-4944

Abstract:

We report a case of acute right ventricular failure in a patient with cardiogenic shock on left-sided mechanical circulatory support with Impella 5.0. The patient was successfully bridged to heart transplantation using additional right-sided support with ProtekDuo. Key learning points of the case include prompt recognition of right ventricular failure in patients on left-sided support, early consideration of right-ventricular mechanical support platforms, and timely deployment of right-sided mechanical support.

Case:

A 61-year-old man with non-ischemic cardiomyopathy and inotrope dependence was admitted for acute cardiogenic shock. Admission vitals and physical exam on his home dose of milrinone $(0.375 \mathrm{mcg} / \mathrm{kg} / \mathrm{min})$ showed the following: blood pressure (BP) $99 / 63 \mathrm{mmHg}$, regular heart rate of 115 beats/min, normal oxygen saturation on room air, jugular venous distention to $14 \mathrm{~cm}$, and cool extremities with significant bilateral lower extremity edema. Notable laboratory findings on initial presentation were creatinine $1.5 \mathrm{mg} / \mathrm{dL}$ (baseline $1.1 \mathrm{mg} / \mathrm{dL}$ ), lactate $0.7 \mathrm{mmol} / \mathrm{L}, \mathrm{LDH} 274 \mathrm{IU} / \mathrm{L}$, normal transaminases and bilirubin. Echocardiogram showed globally reduced systolic function with left ventricular ejection fraction (LVEF) 10-15\%, dilated left ventricle with end-diastolic diameter $7.4 \mathrm{~cm}$, mildly reduced global systolic function of right ventricle (RV) with mild dilation, and RV wall thickness with no thrombus. Given poor response to diuresis and worsening renal function refractory to escalating doses of milrinone, a right heart catheterization (RHC) was performed while the patient was on milrinone $0.50 \mathrm{mcg} / \mathrm{kg} / \mathrm{min}$. RHC was consistent with elevated biventricular filling pressures and low cardiac output (Table 1). An Impella 5.0 was placed for mechanical left-ventricular (LV) support, which resulted in clinical and symptomatic improvement. The patient remained on stable inotropic and mechanical circulatory support over the next several days. Continued hemodynamic monitoring with a pulmonary artery catheter (PAC) showed stable central venous pressures (CVP) of $6-8 \mathrm{mmHg}$ and $\mathrm{PA}$ 
saturations of $55-60 \%$. About a week after Impella placement, he was noted to have an acute increase in his CVP to $30 \mathrm{mmHg}$, a decrease in PA saturation to $36 \%$, cardiac index $1.8 \mathrm{~L} / \mathrm{m}^{2}$, worsening hypoxia, and hypotension (BP 88/59). These hemodynamic changes were preceded by a 37-beat run of ventricular tachycardia followed by spontaneous return to sinus rhythm. Bedside cardiac ultrasound revealed severely reduced RV function. Figure 1 shows the CVP trend leading up to this event.

\section{Additional Medical/Surgical History:}

Implantable cardioverter-defibrillator (ICD) placement, Chronic kidney disease (stage II)

Differential Diagnosis:

The differential for this patient's acute RV failure includes:

- RV infarction

- RV failure precipitated by ventricular arrhythmia

- Acute-on-chronic RV failure precipitated by left-ventricular assist device

- Pulmonary embolism

Investigations:

Laboratory markers of end-organ dysfunction showed an uptrend in serum creatinine and total bilirubin. Chest radiograph revealed worsening bilateral pulmonary edema. From PAC monitoring, the patient's pulmonary artery pulsatility index (PAPI) was determined to be 1.1 (systolic PA pressure $50 \mathrm{mmHg}$, diastolic PA pressure $22 \mathrm{mmgHg}$, CVP $30 \mathrm{mmHg}$ as a surrogate of right atrial pressure).

Management:

Due to acute RV shock, the patient urgently underwent placement of a right-ventricular assist device (RVAD). A Protek Duo extracorporeal pump was placed for RV support. Flow rates of the right- and left-sided devices were adjusted to prevent LV overload. The patient's hemodynamics improved following RVAD placement. The oxygenator was able to be spliced out from the Protek Duo circuit within 2 days of placement of the device, but the patient remained dependent on mechanical circulatory support (MCS) with the Impella and Protek Duo for biventricular failure. The patient was designated Status 1 on the heart transplant listing.

\section{Learning Objectives:}

To recognize the importance of early detection of RV failure through close monitoring of RV hemodynamics in a patient with a left-ventricular assist device.

To consider the options available for percutaneous RV support, and understand the applications and limitations of RV support platforms.

Discussion:

Up to $20 \%$ of patients have RV failure following placement of a LV assist device (LVAD) (1). The mechanisms for this are varied. With left ventricular mechanical support, as cardiac output normalizes, RV preload increases, which can unmask chronic RV dysfunction and manifest as RV shock. Bowing of the interventricular septum towards the LV due to mechanical unloading of the LV can also precipitate RV dysfunction (2). Additionally, ventricular arrhythmias or defibrillator shocks could compromise RV function. In rare cases, embolic phenomena to the coronary or pulmonary vasculature can also result in RV failure, even with therapeutic anticoagulation. In the case presented, the etiology of the patient's acute RV failure was hypothesized to be a result of ventricular arrhythmias or chronic RV failure unmasked by Impella placement. The patient underwent heart transplantation within 5 days of the onset of RV shock, and pathology of the native heart unexpectedly revealed an acute RV infarct from a suspected right coronary artery embolus (Figure 2).

The incidence of biventricular MCS necessitated by RV failure after LVAD placement is reported to be between 9-37\% (3). Early detection of RV failure can be implemented by close hemodynamic monitoring with 
a PAC and using echocardiographic data. In patients with RV shock refractory to initial medical management, early mechanical support with a right ventricular assist device (RVAD) should be considered. The PAPI index has been shown to be an independent predictor of RV failure and need for RVAD implantation in patients on LVADs. A prior study identified a PAPI of $<1.85$ to be a sensitive predictor of RV failure following continuous-flow LVAD placement (4).

Percutaneous RVAD options include venoarterial extracorporeal membrane oxygenation (VA-ECMO), Impella RP, TandemHeart RVAD and Protek Duo. VA-ECMO is an indirect method of bypassing the RV, whereas the other devices directly bypass the RV by providing a RA to PA conduit. VA-ECMO and Protek Duo have the additional benefit of oxygenating capabilities (5) (6). Table 2 summarizes the hemodynamic effects and several of the advantages and limitations of these devices. All these devices come with the risk of bleeding and thromboembolic complications. A distinct advantage of Protek Duo is the ability of this dual-lumen device to be percutaneously introduced through the right internal jugular vein, allowing the patient to remain ambulatory (6). Of note, these devices are for temporary RV support; new technologies for durable RV support remain under investigation.

Follow-Up:

The patient was discharged home after undergoing a heart transplant, and is doing well post-transplantation.

Conclusions:

In this patient, acute RV shock was precipitated by an embolic RV myocardial infarction, leading to biventricular failure requiring dual support with Impella 5.0 and Protek Duo, allowing successful bridging to transplant. This case exemplifies the importance of prompt recognition of RV failure, and selection of appropriate MCS devices for RV support.

References:

1. Ravichandran, A., Baran, D. et al.(2018). Outcomes with the Tandem Protek Duo Dual-Lumen Percutaneous Right Ventricular Assist Device. ASAIO Journal, 64(4), 570-572.

2. Toennes, B., Garan, A.(2016). Percutaneous Right Ventricular Support Devices for Right Ventricular Failure. JACC.

3. Fida N., Loebe M., et al.(2015). Predictors and management of right heart failure after left ventricular assist device implantation. Methodist Debakey Cardiovasc J., 11(1):18-23.

4. Morine K., Kiernan M., et al.( 2016). Pulmonary artery pulsatility index is associated with right ventricular failure after left ventricular assist device surgery. J Card Fail., 22:110-116.

5. Kapur, N., Esposito, M., et al.(2017). Mechanical Circulatory Support Devices for Acute Right Ventricular Failure. Circulation, 136(3), 314-326.

6. Nicolais, C., Suryapalam, M., et al.(2018). Use Of Protek Duo Tandem Heart For Percutaneous Right Ventricular Support In Various Clinical Settings: A Case Series. JACC, 71(11).

List of titles and captions for tables/figures:

Table 1:

Right Heart Catheterization Measurements

Table 2:

Percutaneous Right Ventricular Assist Devices

Figure 1:

Acute sustained rise in CVP leading up to hemodynamic decompensation.

Figure 2: 
2a: Gross examination of the heart showed a small area of hemorrhagic infarction in the lateral wall of the right ventricle, as shown by the arrow.

2b: Microscopic examination showed a hyper-eosinophilic area representing necrotic myocardium surrounded by granulation tissue reaction.

Table 1: Right Heart Catheterization Measurements
\begin{tabular}{|l|l|}
\hline Right Atrial Pressure (RAP) & $22 \mathrm{mmHg}$ \\
\hline Pulmonary Artery Systolic/Diastolic Pressures & $57 / 34 \mathrm{mmHg}$ \\
\hline Mean Pulmonary Arterial Pressure (mPAP) & $45 \mathrm{mmHg}$ \\
\hline Pulmonary Capillary Wedge Pressure (PCWP) & $37 \mathrm{mmHg}$ \\
\hline Transpulmonary Gradient & $8 \mathrm{mmHg}$ \\
\hline Cardiac Output (CO) and Cardiac Index (CI) & Fick: $4.1 \mathrm{~L} / \mathrm{min}, 2.1 \mathrm{~L} / \mathrm{m}^{2}$ \\
\cline { 2 - 2 } & Thermodilution: $3.5 \mathrm{~L} / \mathrm{min}, 1.8 \mathrm{~L} / \mathrm{m}^{2}$ \\
\hline Pulmonary Artery Oxygen Saturation (PA sat) & $65 \%$ \\
\hline
\end{tabular}




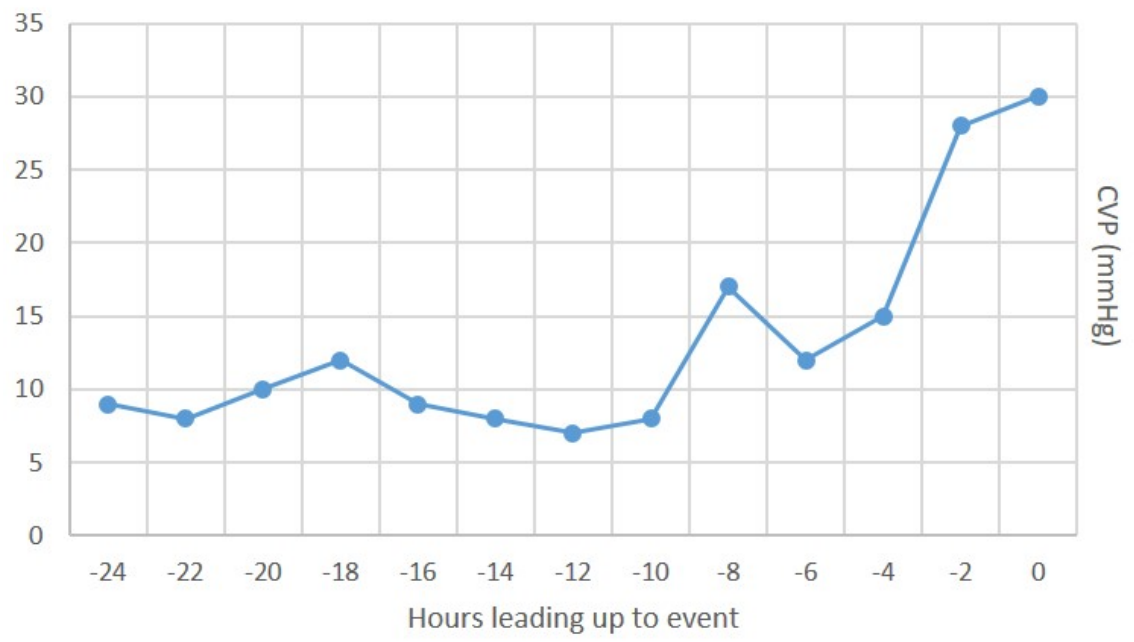



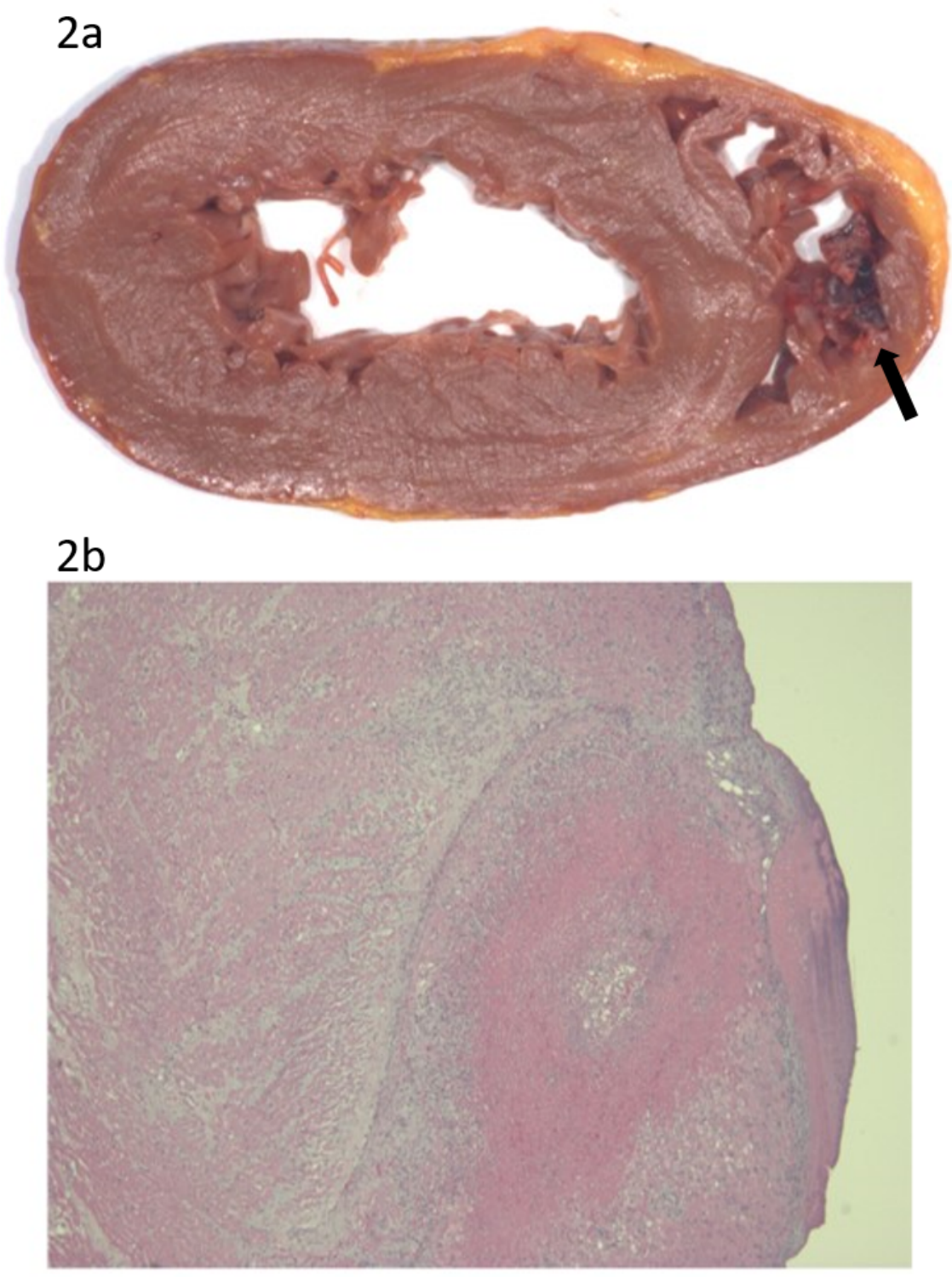


\begin{tabular}{|c|c|c|c|c|}
\hline \multirow{2}{*}{$\begin{array}{l}\text { Percutaneous } \\
\text { RVAD }\end{array}$} & \multirow{2}{*}{\begin{tabular}{|l|} 
Device \\
Characteristics
\end{tabular}} & \multicolumn{2}{|c|}{ Hemodynamic Effects } & \multirow[b]{2}{*}{ Advantages/Limitations } \\
\hline & & Isolated RV failure & Biventricular failure & \\
\hline Impella RP & $\begin{array}{l}\text {-Inflow: RA, } \\
\text { Outflow: PA } \\
\text {-Axial flow } \\
\text {-Flow: } 2-4 \mathrm{~L} / \mathrm{min}\end{array}$ & $\begin{array}{l}\text { RAP: } \downarrow \\
\text { mPAP: } \uparrow \\
\text { PCWP: } \uparrow \\
\text { MAP: minimal effect } \\
\text { Native CO: } \uparrow\end{array}$ & $\begin{array}{l}\text { RAP: } \downarrow \\
\text { mPAP: } \uparrow \\
\text { PCWP: } \uparrow \uparrow \\
\text { MAP: } \uparrow \\
\text { Native CO: minimal } \\
\text { effect }\end{array}$ & $\begin{array}{l}\text { Advantages: } \\
\text { - Direct RV bypass } \\
\text { - RV unloading and } \\
\text { augmentation of native CO } \\
\text { in isolated RV failure } \\
\text { Limitations: } \\
\text { - Large-bore access through } \\
\text { femoral vein } \\
\text { - No oxygenating capacity } \\
\text { - In biventricular failure, in } \\
\text { the absence of LVAD, } \\
\text { increased LV preload }\end{array}$ \\
\hline TandemHeart & \begin{tabular}{|l|}
-Inflow: RA, \\
Outflow: PA \\
-Extracorporeal \\
centrifugal flow \\
-Flow: 2 -4L/min
\end{tabular} & $\begin{array}{l}\text { RAP: } \downarrow \\
\text { mPAP: } \uparrow \\
\text { PCWP: } \uparrow \\
\text { MAP: minimal effect } \\
\text { Native CO: } \uparrow\end{array}$ & $\begin{array}{l}\text { RAP: } \downarrow \\
\text { mPAP: } \uparrow \\
\text { PCWP: } \uparrow \uparrow \\
\text { MAP: } \uparrow \\
\text { Native CO: minimal } \\
\text { effect }\end{array}$ & $\begin{array}{l}\text { Advantages: } \\
\text { - Direct RV bypass } \\
\text { - Oxygenating capacity } \\
\text { - RV unloading and } \\
\text { augmentation of native CO } \\
\text { in isolated RV failure } \\
\text { Limitations: } \\
\text { - Most common cannulation } \\
\text { configuration is using the } \\
\text { bilateral femoral veins } \\
\text { - In biventricular failure, in } \\
\text { the absence of LVAD, } \\
\text { increased LV preload }\end{array}$ \\
\hline ProtekDuo & \begin{tabular}{|l|}
-Inflow: RA, \\
Outflow: PA \\
-Extracorporeal \\
centrifugal flow \\
-Flow: $2-4 \mathrm{~L} / \mathrm{min}$
\end{tabular} & $\begin{array}{l}\text { RAP: } \downarrow \\
\text { mPAP: } \uparrow \\
\text { PCWP: } \uparrow \\
\text { MAP: minimal effect } \\
\text { Native CO: } \uparrow\end{array}$ & $\begin{array}{l}\text { RAP: } \downarrow \\
\text { mPAP: } \uparrow \\
\text { PCWP: } \uparrow \\
\text { MAP: } \uparrow \\
\text { Native CO: minimal } \\
\text { effect }\end{array}$ & $\begin{array}{l}\text { Advantages: } \\
\text { - Direct RV bypass } \\
\text { - Oxygenating capacity } \\
\text { - RV unloading and } \\
\text { augmentation of native CO } \\
\text { in isolated RV failure } \\
\text { - Internal jugular access, } \\
\text { allowing patient to remain } \\
\text { ambulatory } \\
\text { Limitations: } \\
\text { - In biventricular failure, in } \\
\text { the absence of LVAD, } \\
\text { increased LV preload }\end{array}$ \\
\hline VA-ECMO & $\begin{array}{l}\text {-Inflow: RA/venous } \\
\text { system, Outflow: } \\
\text { femoral } \\
\text { arteries/arterial } \\
\text { system } \\
\text {-Extracorporeal } \\
\text { centrifugal flow } \\
\text {-Flow: } 2-6 \mathrm{~L} / \mathrm{min}\end{array}$ & $\begin{array}{l}\text { RAP: } \downarrow \\
\text { mPAP: } \downarrow \\
\text { PCWP: } \downarrow \\
\text { MAP: } \uparrow \\
\text { Native CO: minimal } \\
\downarrow\end{array}$ & $\begin{array}{l}\text { RAP: } \downarrow \\
\text { mPAP: } \uparrow \\
\text { PCWP: } \uparrow \uparrow \\
\text { MAP: } \uparrow \uparrow \\
\text { Native CO: } \downarrow\end{array}$ & $\begin{array}{l}\text { Advantages: } \\
\text { - Cardiopulmonary support } \\
\text { - Oxygenating capacity } \\
\text { Limitations: } \\
\text { - Indirect RV bypass } \\
\text { - Increased LV afterload, } \\
\text { which could require a } 2^{\text {nd }} \\
\text { device for LV unloading } \\
\text { - Large-bore arterial } \\
\text { cannulation }\end{array}$ \\
\hline
\end{tabular}

